La Lectura Rápida en el Desarrollo de la Velocidad y la Comprensión en Inglés. San Juan de Pasto: Universidad de Nariño -CEPUN. ISBN: 978-958-9479-13-1. 1997.

APA citation style: Benavides B., Jorge E. (1997). La Lectura Rápida en el Desarrollo de la Velocidad y la Comprensión en Inglés: desarrollo de la Lectura, Velocidad, Comprensión, Vocabulario y Gramática en Estudiantes de Inglés Técnico a Nivel Universitario. San Juan de Pasto: Universidad de Nariño -CEPUN.

\title{
La Lectura Rápida en el Desarrollo de la Velocidad y la Compresión en Inglés
}

Jorge E. Benavides B. (joelbebu@gmail.com)

Departamento de Lingüística e Idiomas

Universidad de Nariño, San Juan de Pasto, Colombia

\section{Revisión de la literatura}

\subsection{Marco Teórico}

\subsubsection{La velocidad y la comprensión de lectura}

Se podría afirmar, según Nuttall, (1985) que la velocidad de lectura y la comprensión de lectura están muy estrechamente relacionadas a tal punto de decir que una lectura demasiado lenta ofrecería serias dificultades para la compresión. Esto es prácticamente lo que sucede cuando el lector se encuentra en una fase de lectura palabra por palabra lo cual representa problemas para la detección de las ideas y en últimas de la comprensión del significado del texto.

Es también por demás conocido que los estudiantes usualmente tienen un ritmo y velocidad de lectura demasiado bajos no solamente en lengua extranjera sino también en la materna. En nuestros días, caracterizados por la inundación de literatura escrita de toda índole, y en medio de la revolución de la información, necesariamente se tendrían niveles de comprensión y de velocidad mayores que los obtenidos en épocas en las que las formas impresas de la información no estaban lo suficientemente desarrolladas, o como es el caso de las culturas donde la lengua era o todavía sigue siendo oral. Sin embargo, parece que el desarrollo de la velocidad de la lectura no está lo suficientemente desarrollada y enfatizada a nivel curricular y acorde con las enormes cantidades de información escrita en el momento, sobre todo en lengua Inglesa. 
La Lectura Rápida en el Desarrollo de la Velocidad y la Comprensión en Inglés. San Juan de Pasto: Universidad de Nariño-CEPUN. ISBN: 978-958-9479-13-1. 1997.

Las nuevas estrategias metodológicas comunicativas para la lengua extranjera fruto de varias clases de estudios o trabajos tendientes a determinar el desarrollo de la velocidad de la lectura, sobre todo teniendo en cuenta los diferentes propósitos de la misma y por consiguiente los diferentes requisitos y procesos apuntan hacia el manejo natural de la lengua y la metodología comunicativa. Brumffit y Johnson, (1983), por ejemplo, proponen que una de las funciones primordiales de esta metodología es la heurística, es decir, el hecho de utilizar la lengua para aprender y descubrir algo. Aprender algo mediante el manejo apropiado de la lectura en ámbitos específicos en nuestra época es pues una necesidad a nivel académico y profesional. Dada la cantidad de información existente en las diferentes áreas del conocimiento humano, una lectura lenta podría implicar una seria desventaja académica y profesional. Smith, (1982) explícitamente nos propone la cualidad de la lectura de no poderse compartamentalizar en una metodología única, es decir, que la lectura no necesariamente puede enseñarse explícitamente puesto que es una habilidad que se adquiere y se desarrolla leyendo y donde desde al principio el niño empieza un proceso de validación de hipótesis sobre un texto. El papel del profesor tiene que necesariamente acomodarse a esta característica de la lectura en el sentido de que se necesita un guía más que un instructor, buenos materiales con qué trabajar y buena disposición de ambas partes para que el aprendizaje de los diferentes productos colaterales de la lectura se den, es decir, aspectos lingüísticos como la gramática, el vocabulario, y culturales como el conocimiento general y específico.

Muchos estudios se han realizado para desarrollar el proceso de la velocidad de la lectura y la gran mayoría se inclinan por la teoría de que el lector eficiente no lee palabras sino frases, oraciones e ideas (Smith, 1969), y que al hacerlo se está realizando la menor cantidad de fijaciones posibles (movimientos del ojo) en el texto y por ende se va aumentando la velocidad de lectura. Según Smith (1982), las características e implicaciones del proceso psicológico y fisiológico de lectura deben tenerse en cuenta para que el aprendizaje y desarrollo de la lectura se ajusten con las necesidades. Por lo tanto la lectura debe ser:

1. Rápida

2. Selectiva

3. Dependiente de la información no visual.

Tal parece ser que el ojo humano sigue un proceso diferente al de 'ver' o el de 'mirar' cuando leemos un texto donde entre más intentamos 'mirar' menos 'vemos'. Para demostrar esto se han hecho estudios sobre cómo ve el ojo humano cuando leemos y se 
La Lectura Rápida en el Desarrollo de la Velocidad y la Comprensión en Inglés. San Juan de Pasto: Universidad de Nariño-CEPUN. ISBN: 978-958-9479-13-1. 1997.

ha encontrado, por ejemplo, que diez milisegundos sería el tiempo necesario para que el ojo humano pueda recoger información y completar una experiencia perceptual. El mismo tiempo es el que requiere el obturador de una cámara fotográfica para tomar una buena exposición (fotográfica) en condiciones normales de luz. Mucho menos que eso se necesita para que un mensaje del ojo llegue al cerebro.

En cuanto a la velocidad de lectura, parece existir evidencia en el sentido de que leer bien no necesariamente es leer despacio como la asegura el conocido dicho aplicado también a la escritura. La investigación sobre el proceso de lectura, sobre cómo el ojo y el cerebro detectan y utilizan la información visual nos lo aclara Smith al explicar que:

\begin{abstract}
La venerable pieza de equipo psicológico conocido como taquitoscopio, es un dispositivo que presenta la información al ojo por periodos de tiempo bastante cortos. En otras palabras, el taquitoscopio es un dispositivo para estudiar cuánto podemos ver en un solo golpe [...] En su forma más sencilla un taquitoscopio es un proyector de diapositivas que proyecta texto en una pantalla durante una determinada cantidad de tiempo, usualmente una fracción de segundo. Uno de los primeros descubrimientos hechos mediante el uso de este dispositivo fue que el ojo humano tiene que exponerse a la información visual por un tiempo menor que el que generalmente se pensaba. Si hay suficiente intensidad, una exposición de 50 milisegundos es más que adecuada para toda la información que puede manejar el cerebro en cualquier momento dado [...] 50 milisegundos es suficiente exposición para que toda la información visual pueda hacerse con una sola fijación. (p.29)
\end{abstract}

Según estos datos se puede establecer que mirar el texto durante una cantidad de tiempo prolongado no va a mejorar la lectura puesto que el ojo humano y el cerebro no necesitan sino fracciones de segundo para percibir y determinar significado del texto.

Otro descubrimiento importante, según Smith, es el hecho de que al ojo humano, en un proceso de lectura le tomaría el mismo tiempo percibir cinco letras que cinco palabras en una oración con la consiguiente implicación de que a la percepción de letras individuales no tenga que dedicarse demasiado tiempo y que sea un pre-requisito para la percepción y posterior identificación de palabras. Dicho de otra forma, el reconocimiento de palabras no tomaría más trabajo que el del reconocimiento de caracteres individuales. Además, lo que se percibe de una sola y breve presentación depende de lo que se presenta y del conocimiento previo que de eso tiene el sujeto a quien se hace la presentación del texto en la lectura.

Por ende, la velocidad de percepción es directamente proporcional al número de alternativas que el ojo humano tiene que considerar y desechar en el proceso de 
La Lectura Rápida en el Desarrollo de la Velocidad y la Comprensión en Inglés. San Juan de Pasto: Universidad de Nariño -CEPUN. ISBN: 978-958-9479-13-1. 1997.

identificación de la palabra. El ejemplo de Smith es bastante ilustrativo aquí. Podríamos reconocer nuestro carro a cierta distancia más rápidamente entre diez que entre veinte o treinta vehículos, por ejemplo. Y muchísimo más rápido si se hace entre solo cinco. Pero nos demoraríamos muchísimo más si tuviéramos que identificarlo entre cincuenta! En esto radica el contexto de información del proceso de la lectura; es decisivo que utilicemos cualquier aspecto relevante que ya conozcamos, es decir, lo que Smith y otros investigadores llaman información no visual, con el propósito de reducir el número de alternativas.

Otro aspecto importante en el proceso de lectura relacionado con la velocidad es el tipo de visión en el cual se compromete el ojo cuando lee. Se sabe que el ojo humano no se mueve en forma continua como mucha gente, inclusive profesores, creen que sucede al leer un texto. El tipo de movimiento que en realidad nos ocupa para efectos de la lectura es en realidad, como lo explica Smith, un salto rápido, irregular, espasmódico y sorprendentemente preciso que realiza el ojo de un punto a otro del texto. A este salto o movimiento, que sucede como una especie de sacudón o tirón (en inglés se utiliza la palabra francesa saccade) es al que más importancia se da desde el punto de vista de la lectura, fisiológicamente hablando.

En nuestro medio se dice que el ojo humano realiza infinidad de saltos que son guiados por cualquier tipo de impacto o estimulo visual. Las pausas que se hacen entre estos saltos es lo que se denomina una fijación. En el proceso de la lectura, el ojo humano puede leer de izquierda a derecha por medio de saltos y realizando fijaciones entre los mismos. Sin embargo, un lector bastante eficiente haría la menor cantidad de fijaciones en una línea de texto, e idealmente no leería de izquierda a derecha (lectura horizontal) si no que haría una fijación en cada línea lo que determinaría un lectura por líneas, es decir de arriba a abajo (lectura vertical), línea por línea a la vez.

De acuerdo a las investigaciones con el taquitoscopio, durante el salto que hace el ojo al leer, es decir, en el espacio de moverse de un punto a otro, es poco lo que éste ve. En realidad el ojo no recoge la información durante los saltos producidos sino entre las fijaciones o pausas que hace entre los saltos, es decir, cuando el ojo está quieto, cuando se detiene, después de un salto (visión periférica).

El desplazamiento que hace el ojo normalmente desde el extremo izquierdo al extremo derecho de un ángulo de visión de 100 grados es de unos 100 milisegundos, es decir una 
La Lectura Rápida en el Desarrollo de la Velocidad y la Comprensión en Inglés. San Juan de Pasto: Universidad de Nariño-CEPUN. ISBN: 978-958-9479-13-1. 1997.

décima de segundo. Los experimentos con el taquitoscopio demostraron que 50 milisegundos eran suficientes para que el cerebro pudiera con la información en un solo 'golpe', es decir, en un tiempo de exposición suficiente para la información visual que puede obtenerse en una sola fijación. Consecuentemente, de entre tres a cuatro palabras es lo que normalmente se obtiene en una fijación de 50 milisegundos. De esta manera, el número de fijaciones que se hagan en un determinado tiempo depende de la habilidad del lector, de la dificultad del pasaje y del tipo de despliegue del texto (interlineado, columnas, tamaño de las letras, etc.)

El promedio de fijaciones por segundo de un adulto normal puede ser de entre tres a cuatro, sin embargo, lectores más veloces solo lograrían una o dos fijaciones extra por segundo, o sea no existen diferencias dramáticas en cuanto al número de fijaciones por segundo a pesar de que lectores muy rápidos pueden decir que hacen muchas más por segundo. Un adulto normal, en un texto normal de unos 65 a 70 caracteres por línea, es decir, 12 a 14 palabras, realizaría de tres a cuatro fijaciones. Entre mayor sea el número de fijaciones por segundo mayor será la velocidad de lectura. Sin embargo, no se puede entrenar al ojo a que realice más de las fijaciones que puede hacer sin que se afecte la comprensión. No es el hecho de mover el ojo rápidamente sino el abarcar más texto en cada fijación y aumentar el número de fijaciones por segundo y especialmente cuánta información con significado se puede obtener en cada una de ellas.

Lo que distingue a un lector hábil de uno no entrenado adecuadamente es la cantidad de significado que este puede extraer en una fijación. Por consiguiente, con lo expresado por Smith, la mejor forma de evaluar la forma de leer entre dos sujetos es contar el número de fijaciones que se requieren para leer un texto de cien palabras. Los lectores hábiles harán menos fijaciones en cien palabras (abarcando más palabras) que los lectores deficientes ya que los primeros abarcarán más significado en una sola fijación y harán más de estas por segundo. Leer a un nivel menor de 200 palabras por minuto, según Smith conllevaría a tener una visión de túnel. Para evitar la visión de túnel, consistente en cerrar el ángulo de la visión con detalle visual, es necesario que la lectura se haga lo más rápido posible no necesariamente desplazando los ojos con rapidez sino haciendo el menor número de fijaciones posibles. En otras palabras, haciendo uso de la visión periférica, la cual abarca más texto en cada salto, tirón o golpe de visión con la consecuente disminución de número de fijaciones en el texto. 
La Lectura Rápida en el Desarrollo de la Velocidad y la Comprensión en Inglés. San Juan de Pasto: Universidad de Nariño-CEPUN. ISBN: 978-958-9479-13-1. 1997.

Por otra parte, la lectura debería ser rápida especialmente si el objetivo central de una buena lectura es leer tanto texto como sea posible en cada fijación manteniendo el significado, es decir, ampliar el rango de visión desarrollando la visión periférica en el momento en que el ojo se detiene. Esto haría que la lectura sea haga no solo rápida sino efectiva abarcando más significado. La sugerencia habitual de leer despacio cuando no se entienda puede empeorar las cosas y conllevaría a la confusión puesto que se estaría examinando el texto en detalle haciendo del proceso de la lectura uno de palabra por palabra y recurriendo, como se manifestó arriba, a la visión de túnel.

Además, la velocidad de lectura se puede mejorar en forma considerable cuanto menor sea el número de fijaciones en cada línea de texto, desarrollando la visión periférica en lugar de la visión de túnel (lectura palabra por palabra); cuanto menor sea la cantidad de regresiones que tiene que realizar el ojo en una línea, es decir, el hecho de retroceder una o dos fijaciones previas; y cuanto mayor sea el recurso a la información no visual.

Uno de los problemas que se encuentran dentro de la literatura investigativa y dentro de la experiencia docente es que los estudiantes de una lengua extranjera como el inglés usualmente tratan de traducir al mismo tiempo que leen el texto lo cual repercute en su muy bajo nivel de velocidad y por ende en bajos niveles de comprensión. Desafortunadamente, parecería que a los estudiantes no se les enseña a leer a partir de unidades de significado, de ideas completas. Por el contrario, la tendencia es a leer palabra por palabra, recurriendo a la visión de túnel y a la traducción literal viéndose afectados tanto el proceso de comprensión como el de velocidad.

Según Nuttall, (1985), por ejemplo, los ejercicios de skimming y de scanning son los apropiados para desarrollar la habilidad de la lectura rápida y para el desarrollo de la habilidad de percibir unidades de significado cada vez que el estudiante enfrenta un texto. Según la misma autora, los estudiantes de educación secundaria en donde el inglés es una segunda lengua, como en el caso de los inmigrantes en Estados Unidos, el nivel de velocidad de lectura estaría entre 120 y 150 palabras por minuto sin un entrenamiento apropiado. A nivel universitario estos estudiantes estarían leyendo a un promedio inferior de 200 palabras por minuto. Sin embargo, este índice usualmente disminuye cuando los estudiantes tienen que enfrentarse a actividades de estudio con lo cual el problema aumenta. Teniendo esto como un punto de referencia se estima que un hablante nativo del inglés con un nivel de educación e inteligencia normal o promedia, es decir, un 
La Lectura Rápida en el Desarrollo de la Velocidad y la Comprensión en Inglés. San Juan de Pasto: Universidad de Nariño-CEPUN. ISBN: 978-958-9479-13-1. 1997.

estudiante de nivel universitario, debería estar leyendo a razón de aproximadamente 300 a 350 palabras por minuto.

En cuanto al enfoque metodológico de la lectura en el nivel de lengua extranjera se puede destacar principalmente lo concerniente a la concepción comunicativa de la lengua en la cual se destaca el propósito mismo de la lectura y la clase de lectura que se realiza antes de la lectura como tal. De esta manera, leer con un propósito para desarrollar una lectura eficiente implementando técnicas y estrategias de lectura tiene que ver con el aspecto comunicativo de la lengua en lo que toca al aprendizaje de la lengua extranjera. Así, refiriéndose a este tipo de lectura, Nutall (1985) enfatiza las técnicas de scanning (localizar información específica), skimming (determinar la idea general de un texto), lectura receptiva (descubrir en forma precisa lo que el escritor ha querido significar) y lectura responsiva (reflejar lo que el escritor ha querido expresar). En términos generales el aspecto más importante de la lectura es el compromiso e interés del lector en esta actividad. De esta manera, las actividades de comprensión de lectura en si constituyen un paso en una secuencia de etapas que involucran aspectos tales como la motivación, el propósito, el intercambio de la información y la transferencia de la misma.

El uso de materiales auténticos es otra característica del enfoque comunicativo. Este enfoque mantiene que es muy importante brindar a los estudiantes la oportunidad de desarrollar estrategias para comprender la lengua tal como es utilizada por sus hablantes nativos. En otras palabras, la lectura de textos escritos utilizados para divulgar información en cualquier área y por cualquier medio como folletos, revistas, periódicos, libros de lecturas, etc. tiene ese componente de ser fuentes naturales o auténticas (no simplificadas, por ejemplo) de lectura utilizadas por hablantes nativos de esa lengua. En este sentido, Widowson (1979) propone un enfoque más funcional o pragmático para la enseñanza de la lengua cuyo propósito central es el desarrollo de habilidades para "realizar cosas con la lengua", como leer para obtener información y utilizar esa información (transferencia y uso de la información). En otras palabras, en la lectura el 'discurso' como texto escrito es el centro de atención.

\subsubsection{La lectura y el aprendizaje de vocabulario}

Según la reciente evidencia empírica como la obtenida por Herman, Anderson, Pearson y Nagy, (1985) parece que el aprendizaje de vocabulario se realiza por medio de pequeños incrementos, dependiendo de la frecuencia de ocurrencia de un determinado término o elemento léxico en el texto. A todo esto se añade la importancia de la inclusión de 
La Lectura Rápida en el Desarrollo de la Velocidad y la Comprensión en Inglés. San Juan de Pasto: Universidad de Nariño-CEPUN. ISBN: 978-958-9479-13-1. 1997.

información contextual que un término puede representar para el sujeto. Esto refuerza el concepto ampliamente aceptado (también mencionado por Nuttall, 1985) de que se podría incrementar el aprendizaje de vocabulario del inglés incrementando también la cantidad de lectura y de los materiales de lectura.

Varios modelos para el aprendizaje del vocabulario y el desarrollo de la lectura han sido aplicados en el aprendizaje de una lengua extranjera, especialmente aquellos que se basan en el soporte de la información y el conocimiento semántico, ortográfico y lexical como el de Rumelhart (1977) y las nuevas propiedades añadidas a éste mediante el modelo de Stanovich (1980). Los programas basados en estos modelos, brevemente descritos en Mason (1987), utilizan información o significado ortográfico, lexical y semántico en sus trabajos: relativización de oraciones, conjunciones y posesivos en el primero y tanto complementación de oraciones como definición de términos en el segundo.

A pesar de que la información gráfica y textual es particularmente importante en el reconocimiento de vocabulario, la importancia de la información contextual (léxica, sintáctica, semántica y discursiva) y la del conocimiento previo que el sujeto trae al texto (enfatizando por Smith, 1982 como información no visual) hace que el concepto del conocimiento progresivo de vocabulario por medio de pequeños incrementos sea bastante fuerte. Entre más información sobre los elementos léxicos en el texto se provea al usuario, habrá más oportunidades para que éste recoja e integre más significado, y además construya y desarrolle su conocimiento del vocabulario y lo utilice en la lectura. Podríamos decir entonces que el aprendizaje de vocabulario estaría estrechamente asociado (estadísticamente correlacionado) con la cantidad de lectura realizada.

La sugerencia que hace Stanovich (1980) acerca de que el reconocimiento de vocabulario y su posterior aprendizaje no solamente varía con el nivel de proficiencia del lector sino también con la cantidad de aspectos contextuales que rodea una palabra, nos puede dar una idea clara de lo que en realidad implicaría el aprendizaje de vocabulario mediante un programa de lectura guiada. Podemos ver que los modelos de Rumelhart, Smith y Stanovich están bastante relacionados por el hecho de que en el texto existe información tanto gráfica como contextual, y semántica de la cual se sirve el lector siempre y cuando éste tenga un grado aceptable de apego y motivación hacia la lectura. De esta manera, se puede insinuar la estrecha relación que existe entre el aprendizaje del vocabulario 
La Lectura Rápida en el Desarrollo de la Velocidad y la Comprensión en Inglés. San Juan de Pasto: Universidad de Nariño-CEPUN. ISBN: 978-958-9479-13-1. 1997.

(incluida la información gramatical) y la cantidad de exposición e interacción con el texto en la lectura.

Por otro lado, Smith (1982), nos da a conocer tres teorías sobre identificación y reconocimiento de palabras, las cuales tratan de describir de alguna manera la forma como el lector hábil trata de identificarlas al leerlas. La primera de estas se orienta al reconocimiento de la palabra completa, la segunda a la identificación de la palabra mediante sus letras, o sea, reconocimiento letra por letra, y la tercera al reconocimiento hecho mediante amalgamas o grupos de letras. La primera de estas es la más importante a considerar en este estudio puesto que desde este punto de vista teórico su premisa más importante es el hecho de que el lector no se detiene para identificar caracteres o letras individuales para la identificación de una palabra sino la palabra en su totalidad, o sea, en un caso extremo se mantiene como irrelevante la dedicación que se hacer al conocimiento del alfabeto y sus sonidos.

A pesar de que la teoría sobre la identificación y reconocimiento de elementos léxicos o palabras es importante, ésta no parece constituir una respuesta sobre el 'cómo' lo hacemos. La teoría por la cual reconocemos frases o ideas completas más que elementos léxicos aislados, es una de las más aceptadas. Esto da pie para considerar que a mayor grado de exposición del estudiante con los términos del texto en su co-texto, y en un contexto determinado, mayores serán las posibilidades de que éste vaya progresivamente integrando el significado de las palabras a partir de su ocurrencia en el texto y siga construyendo el espectro o gama de significados de las mismas.

Mucho se ha hablado también sobre el muy popular y no tan acertado concepto por el cual la razón de no leer (el no apego a la lectura) se debe al desconocimiento de vocabulario suficiente (sobre todo en lengua extranjera), pero parece que este argumento funciona en forma opuesta, es decir, en realidad no se conoce suficiente vocabulario debido a que no se lee. Según Smith, (1982) y Nuttall, (1985), un lector promedio en su lengua nativa puede reconocer aproximadamente 50.000 palabras. Sin embargo los textos de lectura para estudiantes de lengua extranjera como el inglés, están en el rango de 3.500 palabras en uno de sus niveles altos. Es en este sentido que se podría dirigir el argumento sobre la adquisición de vocabulario: el hablante nativo nunca podría tener un repertorio de 50.000 palabras a nivel de reconocimiento mediante una enseñanza explicita, sino que esto en realidad ocurriría mediante un proceso de exposición a ellas en 
La Lectura Rápida en el Desarrollo de la Velocidad y la Comprensión en Inglés. San Juan de Pasto: Universidad de Nariño -CEPUN. ISBN: 978-958-9479-13-1. 1997.

el texto e interactuar con ellas y su significado en sus diferentes contextos a través una lectura intensiva, continua y sistemática.

Pues bien, este mismo proceso se pretende lograr con estudiantes de inglés como idioma extranjero mediante la lectura guiada de textos auténticos y con un propósito de aprendizaje específico. Por otro lado, este sería un proceso muy diferente del de enseñar vocabulario a través de listas de palabras y mediante el uso del diccionario ya que éste último es solo una ayuda en el proceso y no un medio único y menos un fin. El diccionario (sobre todo bilingüe) no constituiría un texto auténtico ni menos uno que provea el contexto a nivel técnico para el aprendizaje del vocabulario en un área determinada del saber, como el propósito que se persigue en este estudio.

No debemos olvidar que una gran cantidad de vocabulario se aprende mediante la inferencia, la contextualización y el conocimiento previo que el lector aporta en el texto y esto parece ser que se da únicamente a través de la exposición e interacción directa y consistente con el mismo.

\subsubsection{La lectura y el aprendizaje de la gramática}

La evidencia encontrada por Herman, Anderson, Pearson y Nagy (1985), puede aplicarse aquí con respecto al componente de gramática, es decir, este tipo de aprendizaje se realizaría por medio de pequeños incrementos, dependiendo de la frecuencia de ocurrencia de un determinado patrón gramatical como de un patrón o estructura discursiva en el texto. De esta manera, no sería extraño encontrar que como sucede con el aprendizaje de vocabulario se podría incrementar el aprendizaje de aspectos de gramática de la lengua incrementando también la cantidad de lectura y de los materiales de lectura. En otras palabras, la cantidad de exposición e interacción con los patrones gramaticales mediante la lectura es lo que hace que se vaya logrando el aprendizaje de éstos en forma indirecta y progresiva.

La información textual y contextual de la cual puede proveer las bases para que el aprendizaje de la gramática siga un camino similar al del aprendizaje de vocabulario. De la misma manera, la idea de que el aprendizaje se realiza por medio de pequeños incrementos es funcionalmente apropiada puesto que el estudiante procesa la información con diversos propósitos y en diferentes ocasiones donde se asumen la comprensión e interacción con un texto. La comprensión de un determinado texto o partes de él implica el conocimiento de patrones gramaticales que no necesariamente son producto de la instrucción formal. La información no visual de la que habla Smith, (1982) 
La Lectura Rápida en el Desarrollo de la Velocidad y la Comprensión en Inglés. San Juan de Pasto: Universidad de Nariño-CEPUN. ISBN: 978-958-9479-13-1. 1997.

puede aplicarse aquí como el conocimiento que aporta el lector al texto para que este 'tenga' significado y sea comprensible. El aprendizaje de la gramática estaría pues estrechamente ligado (correlacionado estadísticamente) con la cantidad de lectura realizada.

El soporte teórico más importante de los tres aspectos de este trabajo se basa en la hipótesis de adquisición y aprendizaje de la lengua formulada por Krashen (1985) cuando asegura que el lenguaje se adquiere de forma subconsciente a medida que lo utilizamos para propósitos específicos y es consecuentemente diferente al de aprender explícitamente como en el caso de hacerlo solo acerca de la lengua mediante la instrucción directa. Es decir, esto ocurriría cuando no necesariamente enfocamos nuestro esfuerzo en la función de la lengua como tal y en su lugar la utilizamos con propósitos inmediatos a través de un proceso explícito de enseñanza. Krashen sugiere que podemos adquirir la lengua (vocabulario, gramática y aspectos discursivos) mediante la lectura en forma auténtica. Esta clase de actividad se acercaría más, aunque no del todo en el caso de la lengua extranjera, al concepto de adquisición que al de aprendizaje.

\section{Bibliografía}

Brumffit, C. \& Keith Johnson. (1983). The Communicative Approach to Language Teaching. Oxford: Oxford University Press.

Herman, P., R. Anderson, D. Pearson, and W. Nagy. (1985). Incidental Acquisition of Word Meaning from expositions with varied text features. Reading Research Quarterly, 23, 3, 263-84.

Krashen, Stephen. (1985). The Input Hypothesis: Issues and Implications. London: Longman.

Mason, George E. (1987). The Relationship between Computer Technology and the Reading Process: Match or Mismatch? Computers in the School. 4,1, 15-24.

Nuttall, Christine. (1985). Teaching Reading Skills in Another Language. London: Heinemann Educational Books.

Rumelhart, David. (1977). Towards an Interactive Model of Reading. In S. Dornic (ed.), Attention and Performance, VI. pp 573-603. Hillsdale, NJ: Erlbaum.

Smith, Frank. (1982). Understanding Reading. New York: Holt, Rinehart and Winston.

Smith, N. B., \& Strickland, R. G. (1969). Some approaches to reading. Washington: Association for Childhood Education International.

Stanovich, K. E.. (1980). Towards and Interactive-Compensatory Model of Individual Differences in the Development of Reading Fluency. Reading Research Quarterly, 16, 32-71.

Widdowson, H. (1979). The Teaching of English as communication. English Language Teaching. 27.1. 